\title{
STUDY ON RUST DETECTION OF RC STRUCTURE BASED ON ELECTROMAGNETIC PULSED EDDY CURRENT
}

\author{
Jia Jia, ${ }^{*}$ Jun Cheng, ${ }^{* *}$ Yuanheng Zhang, ${ }^{* * *}$ Senhua Zhang, ${ }^{* * *}$ and Xiaogang Li ${ }^{* * *}$
}

\begin{abstract}
A set of rust detecting system for reinforced concrete was designed based on the electromagnetic pulsed eddy current (EPEC). The EPEC detecting test on the reinforced concrete specimens with different reinforcement diameters was performed. The finite element (FE) model was established. The results of the reinforcement position experiment show the Hall voltage peak value (HVPV) of the same lift-off value positively correlates with the reinforcement's diameter, and the HVPV declines fast with the increase of the lift-off value.

The rust experiments results show that with the same liftoff value, the HVPV increases with the increase of the horizontal distance from the Hall sensor to the reinforcement in the specimen. When the Hall sensor is at the same position, the HVPV increases with the increase of the diameter of the reinforcement in the concrete. The Hall voltage (HV), the HVPV, and the time of pulse rising (TOPR) of the same specimen are greater in the rust-free point than in the rusted points. And the results of the numerical simulation verify the validity of the conclusions of the physical experiments. Our above study shows that the detecting method can locate the reinforcement in the concrete accurately and ascertain its diameter effectively. Furthermore, the detecting method can identify the rust region of the reinforcement swiftly and evaluate the rust degree rapidly.
\end{abstract}

\section{Key Words}

Rust degree detection, electromagnetic pulsed Eddy current, reinforced concrete structure, Hall voltage

* Zhejiang Institute of Communications, Hangzhou 311112, China; e-mail: jiajia@zjvtit.edu.cn

** Chongqing Real Estate College, Chongqing 401331, China; e-mail: 591918753@qq.com

*** College of Civil Engineering, Chongqing Jiaotong University, Chongqing 400074, China; e-mail: \{896042732, 799722088, 277955508\}@qq.com

Corresponding author: Jun Cheng

Recommended by Prof. Anmin Zhu

(DOI: 10.2316/J.2019.206-5582)

\section{Introduction}

Reinforced concrete is extensively applied in infrastructure construction [1]. The combination of reinforcement with concrete improves the concrete structure's mechanical property and durability. The concrete protects the reinforcement buried in the concrete from corrosion. The reinforcement still gets rusted. This is the main cause of structural performance degeneration, especially in marine engineering structure [2]. Cooperating with loads and other structural damages, reinforcement rust causes a constant decline in bearing capacity, affecting the structural durability and service life. The tough challenges of structural safety, operation, and maintenance are provided by the rust of the reinforcement [3].

Lacking the detection means for the internal defect of the reinforced concrete structure. Thus, most concrete bridges' safety monitoring proceeds ineffectively. And the bridges' internal rust defects exert more harm on the bridges than the external rust defects. An effective nondestructive rust detecting method is needed to detect the reinforcement's rust.

The existing non-destructive testing (NDT) methods for detecting the rust of the reinforcement buried in the reinforced concrete structure include the analytical methods, the physical methods, and the electrochemical methods. The analytical methods require rational mathematical models. The physical methods, such as ultrasonic testing, infrared thermography, metal magnetic memory method, and X-ray testing, demand complex surface preparation and operation process [4]-[9]. Among the above methods, the electromagnetic method is the simplest one, lacking quantitative judgments for the rust degree and the rust speed of the reinforcement [10], [11].

By measuring parameter changes of metal parts' electromagnetic performance character and metal properties, the EPEC detecting technology can ascertain the damage condition of the metal parts [12]-[15]. Driven by a rectangular wave, the EPEC technique can also measure the aluminium alloys' stress [16]-[18]. 
Existing detecting methods cannot avoid damaging the reinforcement's concrete cover [5]. By using EPEC detecting technology, the non-destructive detecting can be performed without buried transducers. The study of technique's application in rust detecting of reinforcement buried in concrete is carried on [8], [11], [19]. In this paper, the experiments studied the reinforcement rust degree detecting technique for reinforced concrete structure based on one set of EPEC detecting system. The experimental results and the analytical results of the FE model prove the detecting technique's feasibility.

\section{Mechanism of Detection by EPEC}

Normally, a square wave with a certain duty ratio is imposed on the primary coil as the driven signal by the EPEC. With the primary coil, bearing square wave electrical signal, approaching the electrically conductive material or the testing specimen, the interaction in the conductor regenerates transient eddy current and magnetic field. The transient eddy current's size and attenuation are related to the electromagnetic character, geometry, and fitting of the conductor. The electromagnetic receiver can receive information about the testing object's shape and size. In addition, the receiver also can receive the electrical conductivity and the magnetic conductivity of the magnetic field regenerated by the EPEC. By this way, we can accomplish the detection and evaluation on the tested specimen.

For the conductive specimens, skin effect and penetration depth are two important characters for the EPEC density's distribution. The EPEC concentrates on the workpiece's surface close to the coil because of the skin effect, the eddy current' density undertakes exponential decay with the increase of the penetration depth. With the driven signal's frequency increase, the eddy current tends to distribute on the workpiece's surface and the capacity of detecting the minor surface defects increases. Because the high-frequency EPEC drops sharply with the increment of the penetration depth, the defect lying in a certain depth only can generate an ineffective response. On the contrary, the low-frequency EPEC can penetrate deeper into the interior to impact the inner defect while the sensitivity of detecting the surface defect reduces. Thus, the frequency must be chosen appropriately to gain the sensitivity and the detection depth.

As the excitation, the rectangular pulse is entered in both ends of the exciting coil. The pulsed magnetic field $B_{1}$ generated by the exciting coil will force the specimen to induce the EPEC $I_{2}$. Finally, the second magnetic field $B_{2}$ pulsed by the EPEC will interact with the magnetic field $B_{1}$ opposite to $B_{2}$. Then, it will change the original magnetic field, the magnetic flux of the coil, and the impedance of the coil. If the specimen under test has flaws or defects, the distribution of EPEC will be influenced. The magnetic field's change influenced by the change of the EPEC will change the induced voltage in the coil under test. By analysing the induced voltage in the coil, we can obtain the information about the specimen's flaws and defects. In this paper, the magnetic measurement sensor (Hall sensor) was

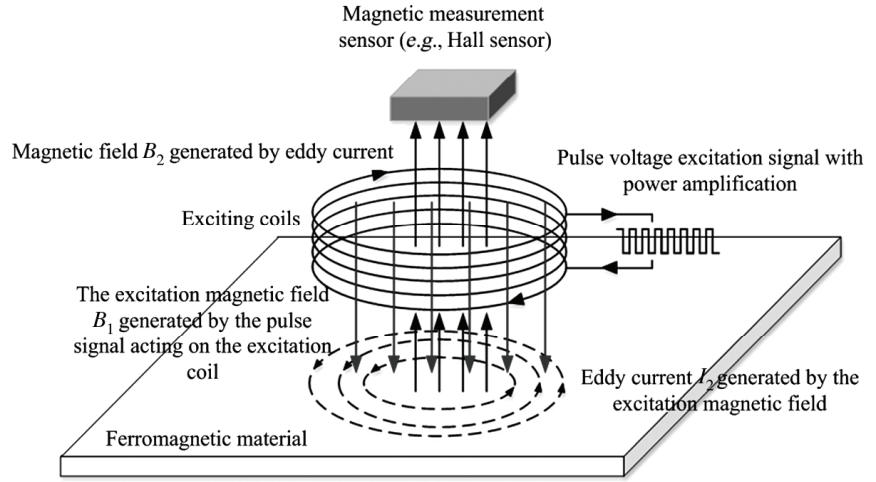

Figure 1. The basic work principle of EPEC based on detection by Hall sensor.

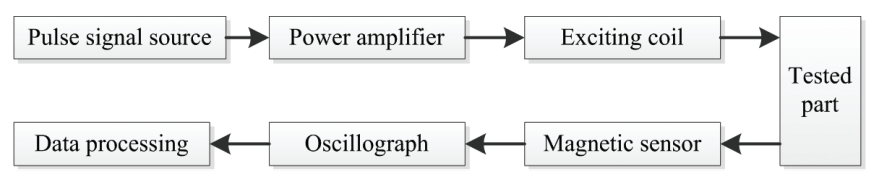

Figure 2. EPEC detecting system frame.

used to detect the magnetic field generated by the EPEC. The detailed work principle is shown in Fig. 1.

\section{Experiment on Detection by EPEC}

\subsection{The Design of the Detecting System}

Figure 2 shows the EPEC detecting system used in the given experiments. The system consisted of the pulse signal source, a power amplifier, an exciting coil, a magnetic sensor, an oscilloscope, and the specimen.

The power amplifier amplified the exciting signal generated by the pulse signal generator. And the pulse signal was input into both ends of the exciting coil to generate the exciting pulse magnetic field. Then, the specimen generated an eddy current magnetic field. Then, the specimen generated an eddy current magnetic field under the effect of the exciting pulse magnetic field. Placed at the bottom of the exciting coil's centre, the magnetic sensor can receive the signal from the eddy current magnetic field generated by the specimen, the exciting coil, and the magnetic sensor constitute sensor probe. After the oscilloscope converted the electrical signal into an image, the data analysis and the defect identification were performed.

In this system, the oscilloscope was serial FY2200S and the power amplifier was DDS function signal producer. The exciting coil winded 500 times by 0.3 -mm-coated encased copper wires owned $10 \mathrm{~mm}$ inner diameter and $22 \mathrm{~mm}$ outer diameter. And the magnetic sensor was highly sensitive Hall sensor module of type SS495A. In addition, the oscilloscope was digital storage oscilloscope of type TDS2012C, the product of Tektronix company, USA.

\subsection{The Experiment Program Design}

In the experiment, the experiment platform set up in Section 3.1 was applied to detect the rusted parts of 


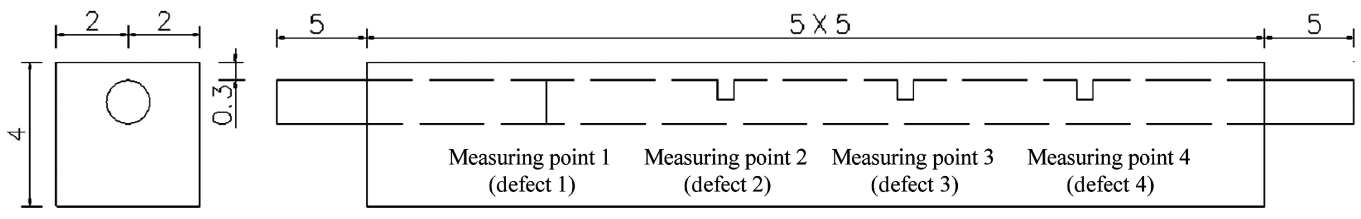

Figure 3. Schematic drawing of element dimension of the specimen and layout of measure points (unit: $\mathrm{cm}$ ).

Table 1

The Number Arrangement of the Specimen

\begin{tabular}{|c|c|c|}
\hline $\begin{array}{l}\text { Reinforcement } \\
\text { Diameter (mm) }\end{array}$ & $\begin{array}{l}\text { Artificial } \\
\text { Simulating }\end{array}$ & $\begin{array}{l}\text { Electrical Chemical } \\
\text { Corrosion }\end{array}$ \\
\hline$\Phi 10$ & Specimen 1 & Specimen 3 \\
\hline$\Phi 16$ & Specimen 2 & Specimen 4 \\
\hline
\end{tabular}

Table 2

The Parameter of Local Rust Amount of the Reinforcements in the Artificially Simulating

\begin{tabular}{|l|c|c|c|c|}
\hline Description & $\begin{array}{c}\text { Rust I } \\
(0 \mathrm{~mm} \times 0 \mathrm{~mm})\end{array}$ & $\begin{array}{c}\text { Rust II } \\
(3 \mathrm{~mm} \times 5 \mathrm{~mm})\end{array}$ & $\begin{array}{c}\text { Rust III } \\
(3 \mathrm{~mm} \times 3 \mathrm{~mm})\end{array}$ & $\begin{array}{c}\text { Rust IV } \\
(5 \mathrm{~mm} \times 3 \mathrm{~mm})\end{array}$ \\
\hline Width $(\mathrm{mm})$ & 0 & 3 & 3 & 5 \\
\hline Depth $(\mathrm{mm})$ & 0 & 5 & 3 & 3 \\
\hline
\end{tabular}

Table 3

Rust Time of Different Rust Stages of the Electrochemical Corrosion

\begin{tabular}{|l|c|c|c|c|}
\hline Description & $\begin{array}{c}\text { Stage I } \\
(0 \mathrm{~h})\end{array}$ & $\begin{array}{c}\text { Stage II } \\
(20 \mathrm{~h})\end{array}$ & $\begin{array}{c}\text { Stage III } \\
(40 \mathrm{~h})\end{array}$ & $\begin{array}{c}\text { Stage IV } \\
(60 \mathrm{~h})\end{array}$ \\
\hline Rust time $(\mathrm{h})$ & 0 & 20 & 40 & 60 \\
\hline Current $(\mathrm{A})$ & $/$ & 0.5 & 0.5 & 0.5 \\
\hline
\end{tabular}

the reinforcements and the rust degree. The artificial defects and electrochemical corrosion defects were used to simulate different rust degrees of the different diameter reinforcements in the specimens. The diameters of the reinforcement are shown in Fig. 3, and the experiment scheme is shown in Table 1.

Figure 3 illustrates each specimen's measure points. The artificial rust points included one rust-free point (point 1) and three rusted points. The points 2 and 3 were regions with the same rust width but different rust depths. The points 3 and 4 were regions with the same rust depth but different widths. The points 2 and 4 were regions with the same rusting volume. Detail sizes of defects are shown in Table 2. The electrochemical corrosion based on the electrochemical reaction was processed with constant current $(0.5 \mathrm{~A})$. The rust rime of different electrochemical corrosion processed was divided into four rust stages. Detailed rust time of different rust stages is shown in Table 3.

\subsection{The Experimental Process}

Before casting the concrete, the experiment on detecting the position of the reinforcement was carried out. The reinforcements with two diameters were scanned along the direction, perpendicular to the reinforcement length, horizontally and respectively. The lift-off value of the experiment was 1,3 , and $5 \mathrm{~mm}$. After the 28-day concrete curing, the electrochemical corrosion of the specimen 3's and 4's reinforcements was tested. The DC stabilised power supply provided the constant current $(0.5 \mathrm{~A})$ to speed up the specimen's corrosion. The four measure points were detected when each rust stage was completed.

In the experiment, the plus-minus bipolar square wave, with $20 \mathrm{~Hz}$ exciting frequency, $\pm 3 \mathrm{~V}$ exciting voltage peakpeak, $50 \%$ pulse square wave duty ratio, was employed as the exciting signal. The Hall sensor was placed close to the specimens with different lift-off values to detect the measure points. The pulse, the HV, and the HVPV were collected by the LabVIEW software.

\subsection{Experimental Results}

\subsubsection{Results of Reinforcement Position Experiment}

The vertical HVPV of naked reinforcements is shown in Figs. 4 and 5.

Hall sensor approaching the reinforcement, the HVPV rose. When the sensor with three different lift-off values 


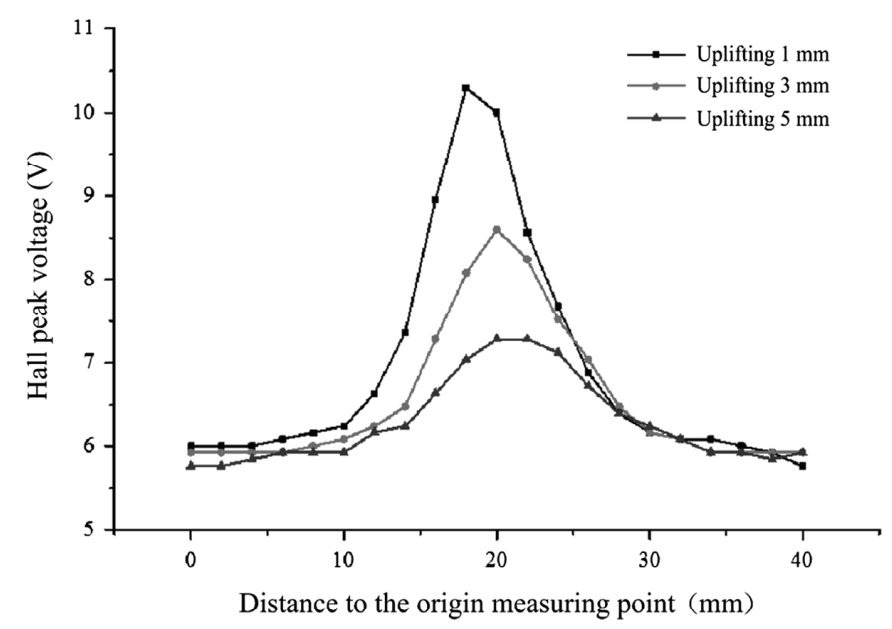

Figure 4. Positing $\Phi 10 \mathrm{~mm}$ reinforcement with pulse square wave.

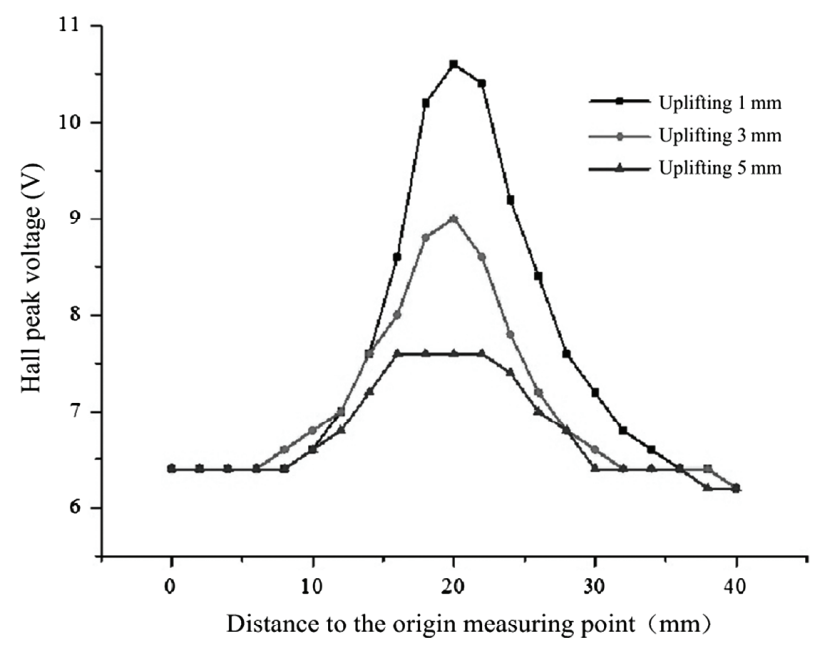

Figure 5. Positing $\Phi 16 \mathrm{~mm}$ reinforcement with pulse square wave.

Table 4

The Hall Voltage Peak Value When Hall Sensor was Right above the Reinforcement (Unit: V)

\begin{tabular}{|c|c|c|c|}
\hline $\begin{array}{l}\text { Lift-Off Value }(\mathrm{mm}) \\
\begin{array}{l}\text { Reinforcement } \\
\text { Diameter }(\mathrm{mm})\end{array}\end{array}$ & 1 & 3 & 5 \\
\hline$\Phi 10$ & 10.3 & 8.6 & 7.3 \\
\hline$\Phi 16$ & 10.6 & 9.0 & 7.6 \\
\hline
\end{tabular}

shifted to right above the reinforcement, the HVPV reached a maximum value as shown in Table 4 . The sensor moving away from the reinforcement, the HVPV dropped. Thus, with certain lift-off value, the detecting system can detect the reinforcement's position.

According to Table 4, the reinforcement with larger diameter owns a greater HVPV with the same lift-off value and the difference is significant enough to identify the reinforcement's diameter. The HVPV declines fast with

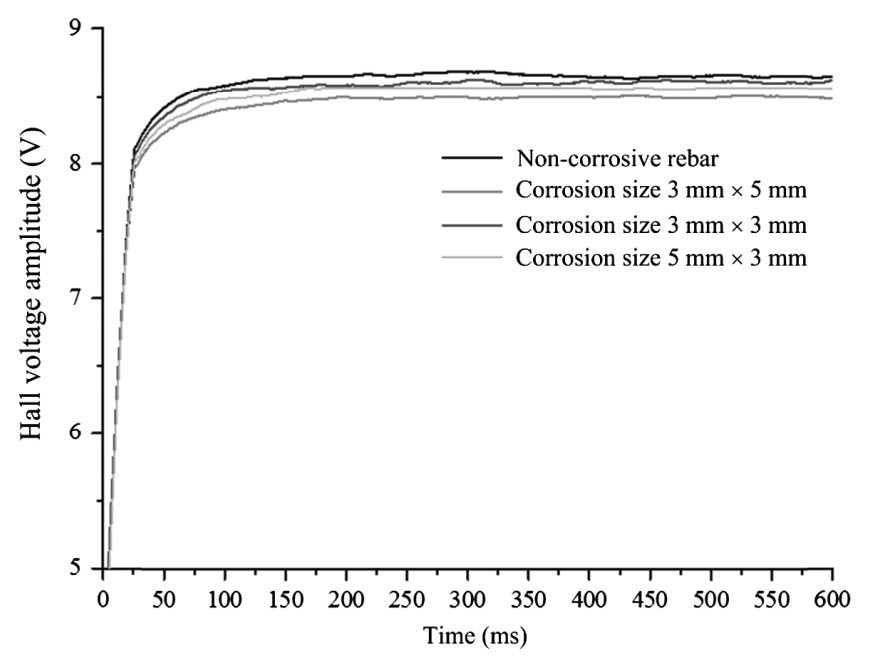

Figure 6. The HV curve of all checking points of specimen 1 .

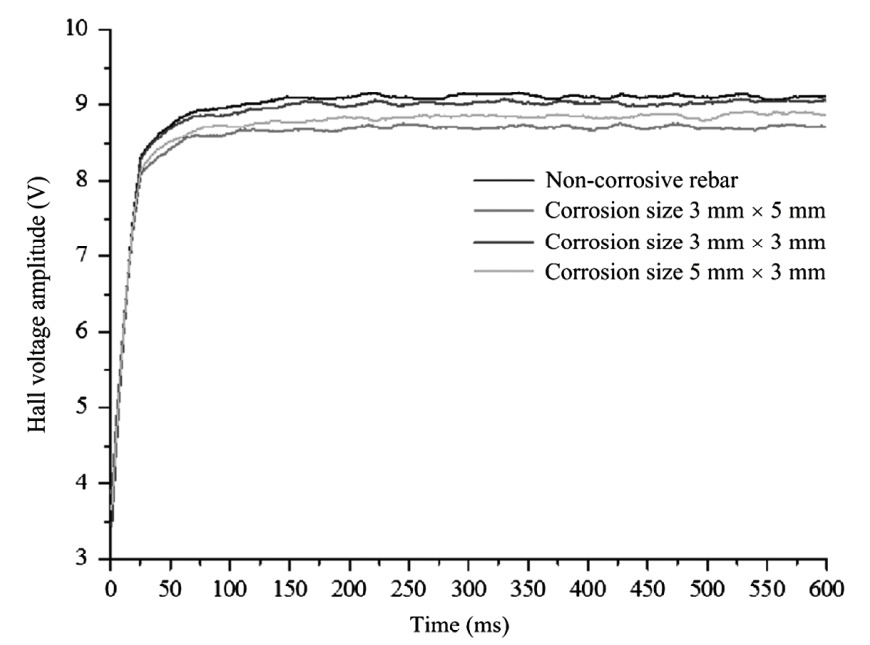

Figure 7. The HV curve of all checking points of specimen 2 .

the increase of the lift-off value. Hence within appropriate lift-off distance, the detecting system can distinguish the reinforcement's size.

\subsubsection{Artificial Rust Experiment}

Figures 6-9 show the HV signals measured at four checking points of specimens 1 and 2 within half cycle. According to Figs. 6-9, the TOPR and the HV including the HVPV of the rust-free parts are longer and greater than the rusted parts. Comparing rust II with rust III (with same width but different depth), the deeper is the rust, the smaller is the HV, the smaller is the HVPV, and the shorter is the TOPR. Comparing rust III with rust IV (with same depth but different width), the wider is the rust, the smaller is the HV, the smaller is the HVPV, and the shorter is the TOPR. Comparing rust II with rust IV (with same rust volume), the $\mathrm{HV}$, the HVPV, and the TOPR of rust II is smaller than that of rust IV, which means that the depth of the rust exerts a greater impact on the signals. 


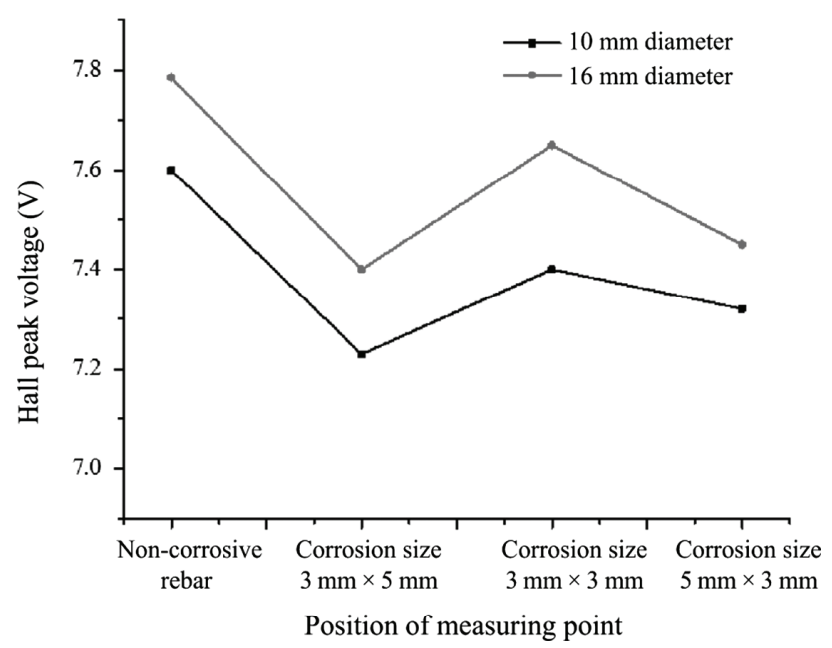

Figure 8. HVPV of all checking points of specimens 1 and 2.

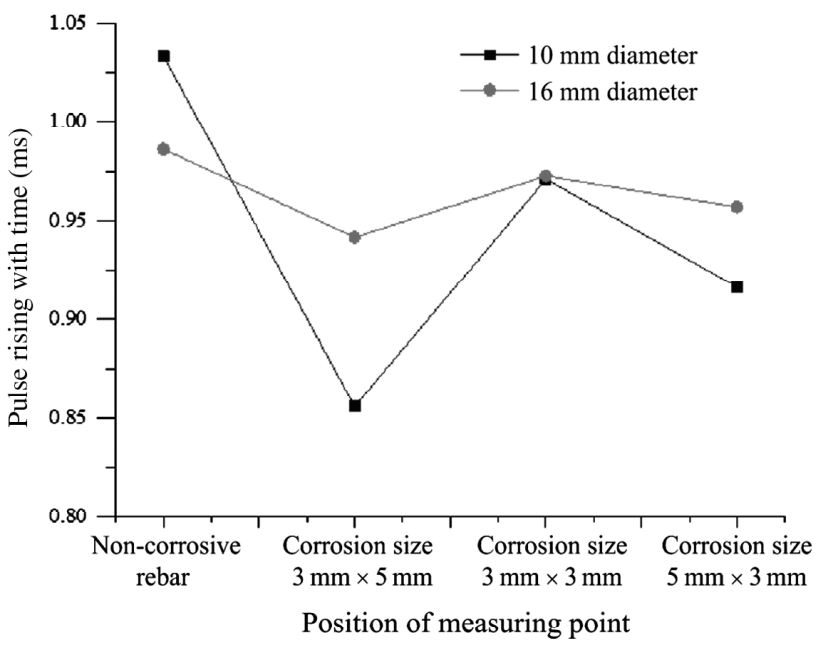

Figure 9. TOPR of all checking points of specimens 1 and 2 .

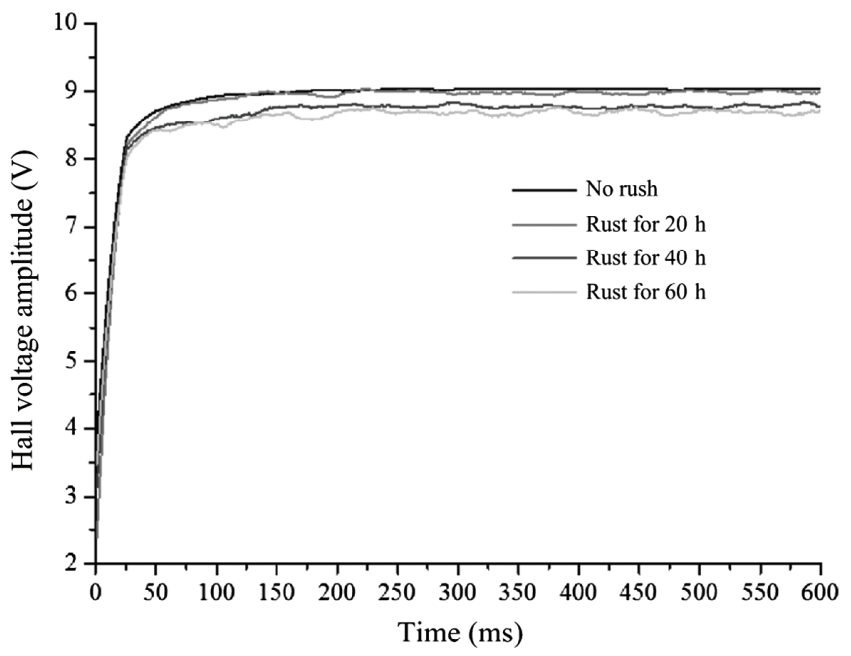

(a)
As shown in Figs. 7-9, the HV and the HVPV decline with the increase of the specimens' corrosion. The decline is more sensitive to the rust's depth than its width. The greater is the diameter of the reinforcements, the greater are the HV and the HVPV. Thus, the HV and the HVPV can identify reinforcement's diameter and the corrosion situation. Moreover, they are more effective to detect the deeper corrosion rather than the wider one. As shown in Fig. 9, the TOPR can also be used to detect the corrosion situation of the reinforcement in the concrete, and it is more effective to detect the deeper corrosion rather than the wider one, while the diameters of the reinforcements cannot be identified by it.

\subsubsection{Electrochemical Rust Experiment}

The HV within half cycle measured at the measuring points of the specimen at different rust stages 3 and 4 are shown in Figs. 10-12.

As shown in Figs. 10-12, the specimens' rust time increasing, the reinforcement's rust degree increases, the reinforcement's effective section decays. In the meantime, the HV declines, the HVPV decreases and the TOPR becomes shorter. Identical to the results of the artificial rust experiment, the HV, the HVPV, and the TOPR can detect the corrosion degree of the reinforcement within the concrete cover.

In conclusion, the results of artificial and electrochemical rust experiment show that with the same lift-off value, the HVPV increases with the increase of the horizontal distance from the Hall sensor to the reinforcement. Thus, this detecting system can locate the position of the reinforcement in the concrete.

When the Hall sensor is at the same position, the HVPV increases with the increase of the reinforcement's diameter, justifying the detecting system can identify the reinforcement's diameter. The HV, the HVPV, and the TOPR of the same specimen are greater in the rust-free

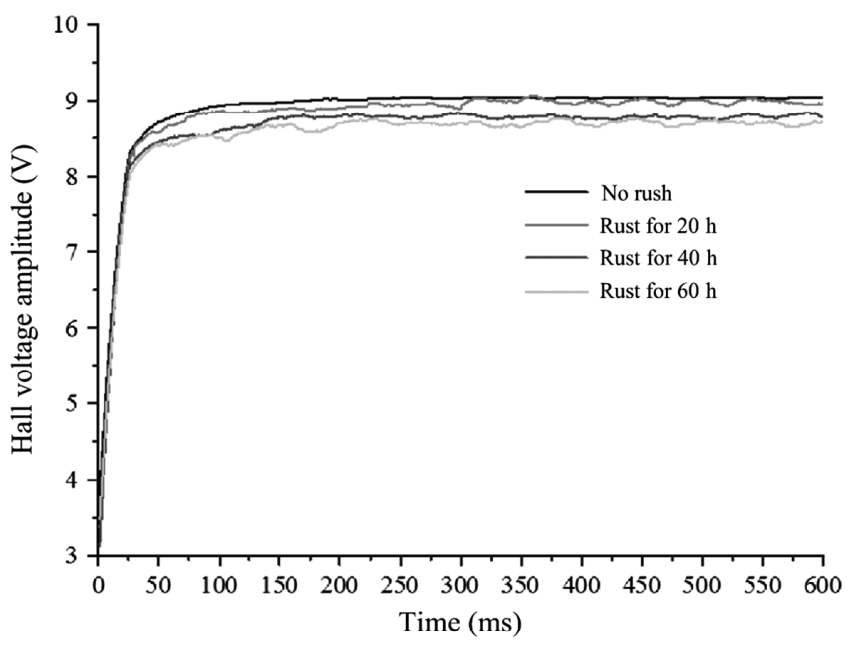

(b)

Figure 10. The HV curves at checking points I and II of specimen 3 at different rust stages. 

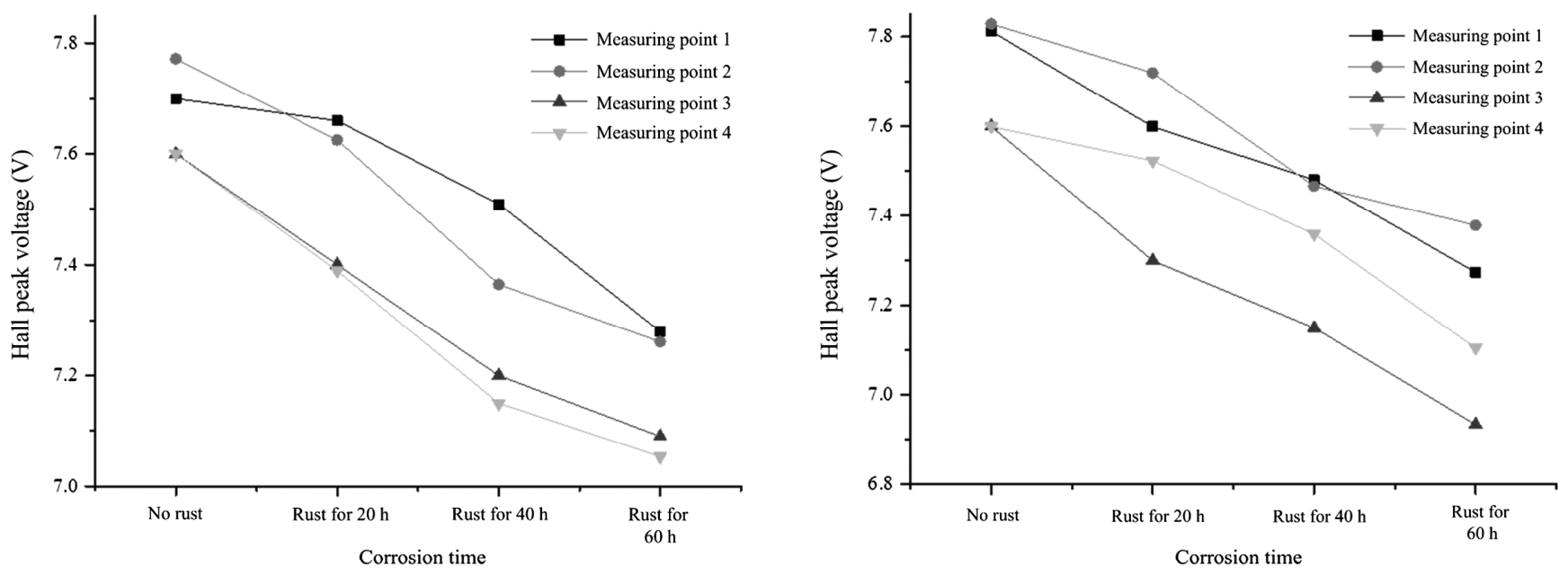

Figure 11. The HVPV at all checking points of specimens 3 and 4 at different rust stages.
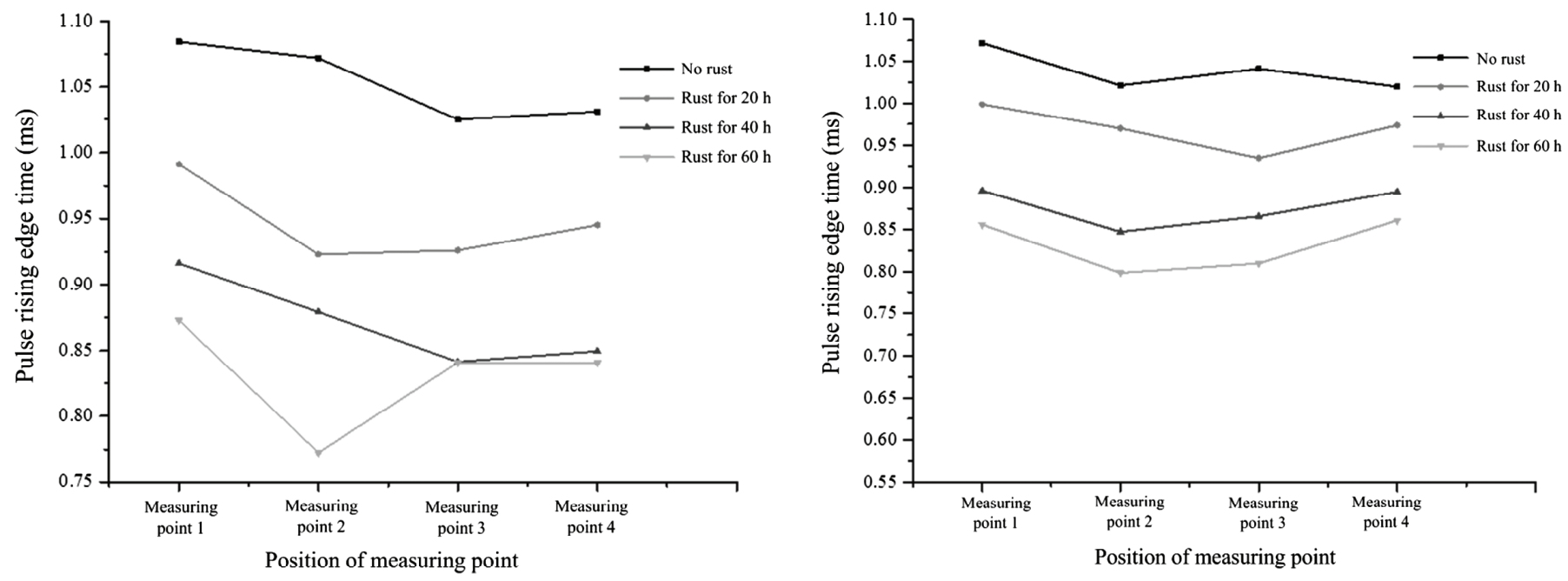

Figure 12. TOPR at all checking points of specimens 3 and 4.

point than in the rusted point, justifying the detecting system can ascertain the reinforcement's rust area. In addition, this given method can be used to evaluate the reinforcement's rust degree, because of the HV, the HVPV, and the TOPR increase with the increase of the amount of the rust reinforcement's rust.

\section{The Finite Element Simulating Analysis}

The FE model was established to simulate the rust degree detecting for the reinforcement in the concrete component using EPEC. With certain thick concrete cover, the simulating results with different rust degrees were collected to compare with the results of previous experiments.

The simplified 2D plan model was derived from the 3D model, and half symmetrical structure was taken to process transient analysis. Without considering the effect of the displacement current, the magnetic vector potential method was used to solve the problems of $2 \mathrm{D}$ or $3 \mathrm{D}$ magnetic field containing eddy current field, while the accuracy of this method will be affected by the magnetic conductive materials.

1. Material: the relative magnetic conductivities of concrete and air were 1 . The relative magnetic conductivity of the exciting coil $\mu_{r}=1$; resistance rate $\rho=1.71 \times 10^{-8} \Omega \mathrm{m}$. The relative magnetic conductivity and the resistance of reinforcement varying greatly with its temperature, the relative magnetic conductivity was 200 , and resistance $\rho=1.71 \times 10^{-8} \Omega \mathrm{m}$; in this paper, because the EPEC was not significant enough to change the temperature of the reinforcement obviously during the experiment.

2. Element: the PLANE53 element was used to simulate the concrete, coil, steel, and air. And the INFIN110 element was used to simulate the far-field air. The CIRCU124 element was employed to simulate the resistance and voltage source.

3. Boundary conditions: the parallel boundary condition of flux in the vicinity of the plane region was applied and the outer wear layer of the air was specified in 


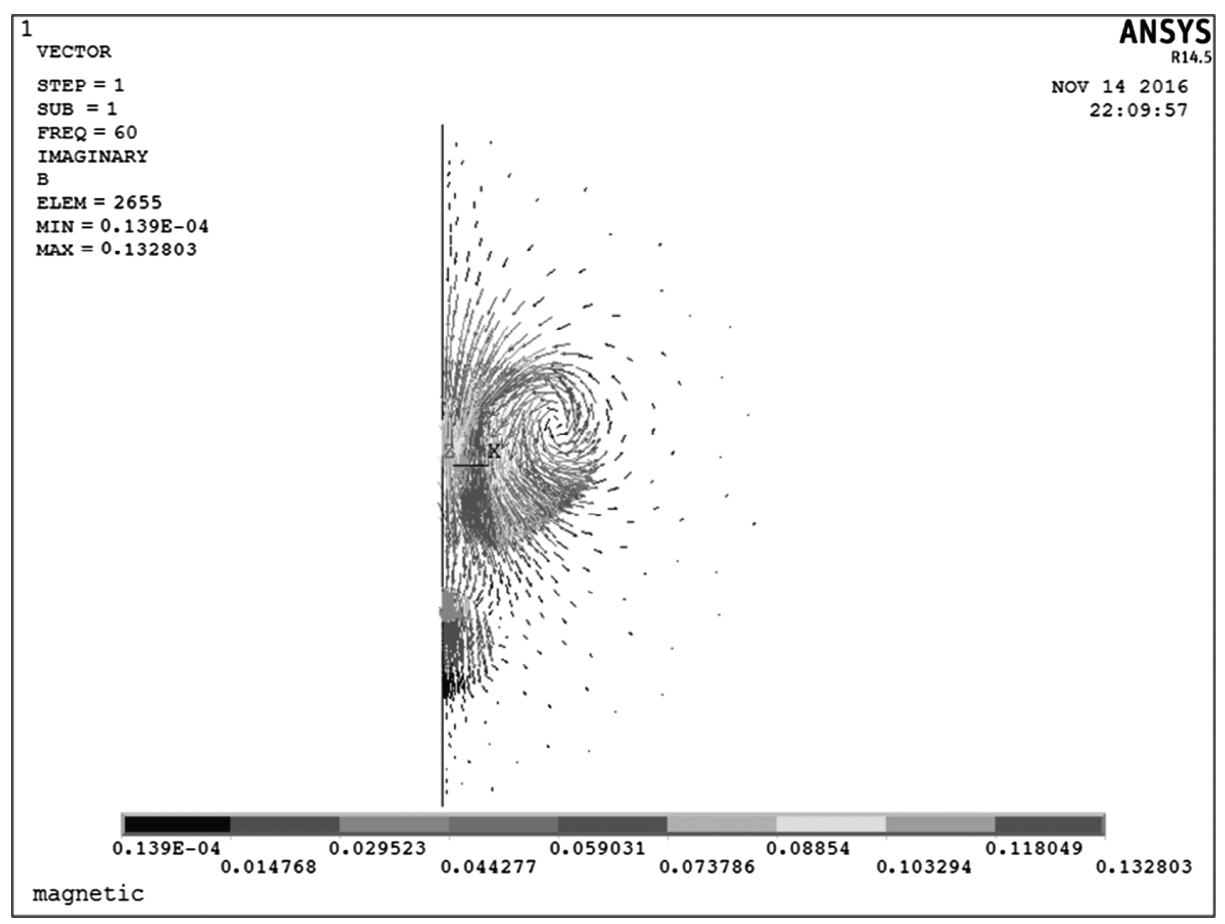

Figure 13. Magnetic flux density drawing.

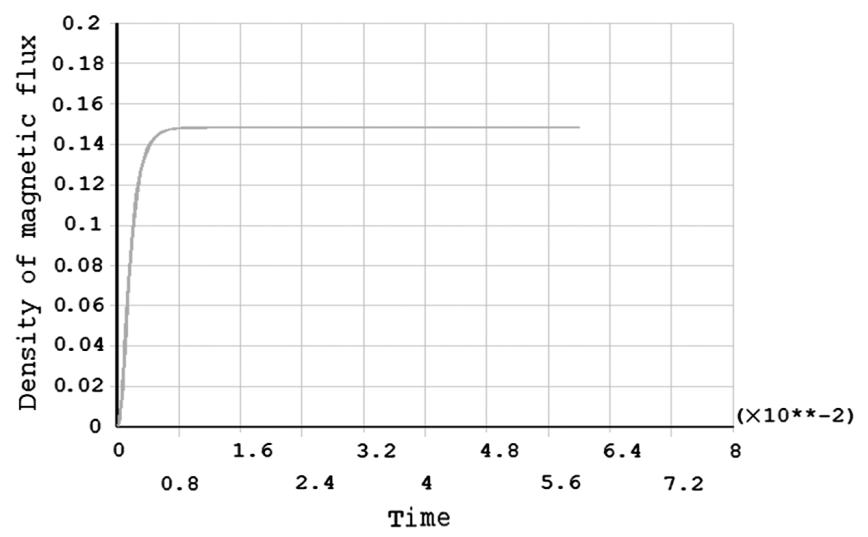

Figure 14. Magnetic flux density changing in the $Y$-direction of the centre of the sensor.

the model based on the Dirichlet boundary condition. And the electrical potential was defined as 0 for the remote airfield with 12 times coil diameter to certify the calculation accuracy.

4. Load: the coupled circuit defined by element CIRCU124 was used to connect exciting pulse voltage square wave to excite the coil in cooperation with the PLANE53 element.

As shown in Fig. 13, the magnetic field distribution around the reinforcement under pulse eddy current sensor can be obtained by numerical analysis. The magnetic flux density at the centre of the coil was in the $Y$-direction, vertical to the surface of the specimens under test, and reached the highest, justifying the correct position of the PEC sensor and the distribution of magnetic radiation.

As shown in Fig. 14, the FE method was applied to simulate the law of magnetic flux density in the $Y$-direction

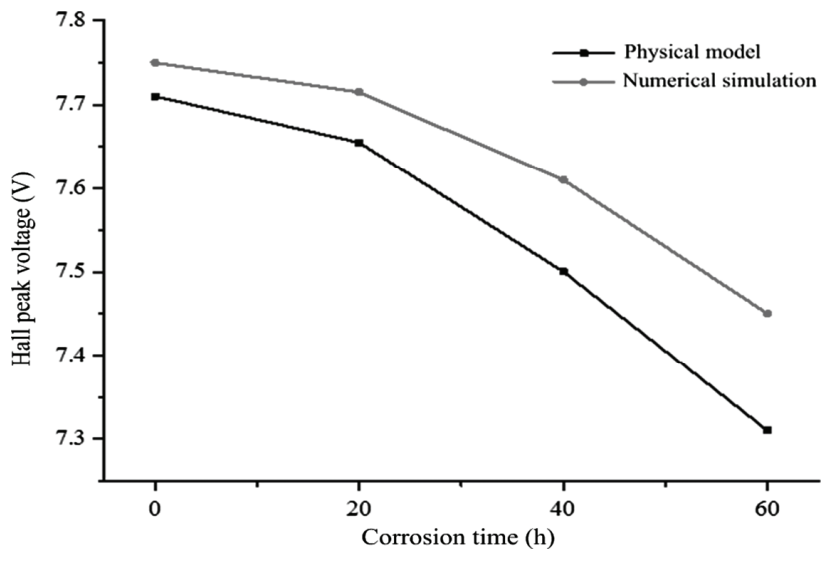

Figure 15. Hall peak voltage with different rust degrees and certain lift-off value $(3 \mathrm{~mm})$.

(By) based on the effect of PEC. The node was set at the centre point of the Hall magnetic sensor, and the results agreed with the experiment results.

Collected from numerical simulate and physical experiments by Hall sensor whose lift-off value is $3 \mathrm{~mm}$, the voltage peak value of the reinforcement with different corrosion degrees is shown in Fig. 15.

The results of the FE model are identical to the physical experiments, but there are still some differences between them. The reason is that the FE model used the magnetic conductivity reduction method to describe the behaviour of the rusted reinforcement under the effect of EPEC. Moreover, it is hard to measure the corrosion products, because the corrosion products may be different due to the corrosion environment. Nevertheless, the results of the FE model and physical experiments both show that the HVPV decreases with the increase of the rust. Hence, the EPEC method can detect the reinforcement's rust degree. 


\section{Conclusion}

This paper presented a set of independently designed rust detecting system based on the working principle of PEC. The physical experiments were carried out after the specimens were prepared. In the experiment, this given detecting system was applied to detect specimens with different rust defects. And the FE model was established to verify the results of the physical experiment.

Main conclusions of this study are exhibited as follows:

1. A set of rust detecting system based on EPEC has been designed and established independently. This system was applied to conduct the experiments on detecting the rust defects of the reinforcements with different diameters in the reinforced concrete; the results of the experiments manifesting this given detecting system can locate the reinforcement and ascertain the diameter of the reinforcement buried in the concrete.

2. The rust degree of the reinforcement with artificial rust defects or electrochemical rust defects was determined by this given detecting system in the experiments. The results show that this given detecting system can be employed to evaluate the rust degree of the reinforcement in the concrete.

3. The results of the numerical simulation by the $\mathrm{FE}$ model verifies the validity of the conclusions of the physical experiments. The results of the numerical simulation verify this given detecting system based on EPEC can be applied to locate the reinforcement in the concrete, to ascertain the diameter of the reinforcement in the concrete, to identify the rust region swiftly, and to evaluate the rust degree.

\section{Acknowledgement}

This work was supported by the National Key Research and Development Program of China (2016YFC0802202), the National Science Fund for Distinguished Young Scholars (51425801), the Major Topic Special Key Research and Development Project of the Artificial Intelligence Technology Innovation in Chongqing (cstc2017rgzn-zdyfX0029), the Science and Technology Planning Project of Yunnan Province of China (2017IB025), the Science and Technology Project of Guizhou Provincial Transportation Department (2016-123-006), and the Science and Technology Planning Project of Nanjing of China (201727002).

\section{References}

[1] C.A. Apostolopoulos and V.G. Papadakis, Consequences of steel corrosion on the ductility properties of reinforcement bar, Construction and Building Materials, 22(12), 2008, 2231622324 .

[2] H. Zhang, J.T. Zhou, R.Q. Zhao, et al., Experimental study on detection of rebar corrosion in concrete based on metal magnetic memory, International Journal of Robotics and Automation, 2017, 32(5), 530-537.

[3] B.A. Lepine, B.O. Wallace, D.S. Forsyth, et al., Pulsed eddy current method developments for hidden corrosion detection in aircraft structures, Defence and Civil Institute of Environmental Medicine, 20(6), 1998, 6-15.

[4] M. Kurodam, S. Yamanaka, and Y. Isobe, Detection of plastic deformation and estimation of the maximum value of residual stress in low carbon steel by x-ray stress analysis using statistical techniques, NDT $\&$ E International, 36(7), 2003, 497-502.

[5] K. Kobayashi and N. Banthia, Corrosion detection in reinforced concrete using induction heating and infrared thermography, Journal of Civil Structural Health Monitoring, 1(1-2), 2011, 25-35.

[6] A.R. Clough and R.S. Edwards, Characterisation of hidden defects using the near-field ultrasonic enhancement of lamb waves, Ultrasonics, 59, 2015, 64-71.

[7] R. Kaushik, J.Z. Xiao, S.L. Joseph, et al., Polygon-based 3D scan registration with dual-robots in structured indoor environments, International Journal of Robotics and Automation, 27(1), 2012, 101.

[8] X.G. Li, J.T. Zhou, L.Y. Zhang, et al., Automatic monitoring of continuous rigid frame bridges by a magneto-elastic effect method, International Journal of Robotics and Automation 32(1), 2017, 41-47.

[9] Y.J. Li, Q.Y. Liu, Y.H. Chen, et al., Helical-contact deformation measuring method in oil-gas pipelines, International Journal of Robotics and Automation, 32(1), 2017, 55-62.

[10] Y. Li, B. Yan, D. Li, et al., Pulse-modulation eddy current inspection of subsurface corrosion in conductive structures, NDT \& E International, 79, 2016, 142-149.

[11] Y.Z. He, G.Y. Tian, H. Zhang, et al., Steel corrosion characterization using pulsed eddy current systems, IEEE Sensors Journal, 12(6), 2012, 2113-2120.

[12] J.C. Moulder, M.W. Kubovich, E. Uzal, et al., Pulsed eddycurrent measurements of corrosion-induced metal loss: Theory and experiment, Review of Progress in Quantitative Nondestructive Evaluation (USA: Springer, 1995).

[13] Y.Z. He, Y.T. Gui, M.C. Pan, et al., An investigation into eddy current pulsed thermography for detection of corrosion blister, Corrosion Science, 78(1), 2014, 1-6.

[14] R.Z. Yang, Y.Z. He, B. Gao, et al., Lateral heat conduction based eddy current thermography for detection of parallel cracks and rail tread oblique cracks, Measurement, 66(1/2), 2015, 54-61.

[15] Y.C. Ou and N.D. Nguyen, Influences of location of reinforcement corrosion on seismic performance of corroded reinforced concrete beams, Engineering Structures, 126, 2016, 210-223.

[16] M. Morozov, G.Y. Tian, and D. Edgar, Comparison of PEC and SFEC NDE techniques, Nondestructive Testing and Evaluation, 24(1-2), 2009, 153-164.

[17] Y.Z. He, F.L. Luo, M.C. Pan, et al., Pulsed eddy current technique for defect detection in aircraft riveted structures, NDT \& E International, 43(2), 2010, 176-181.

[18] M. Morozov, G.Y. Tian, and P. J. Withers, The pulsed eddy current response to applied loading of various aluminium alloys, NDT \& E International, 43(6), 2010, 493-500.

[19] J.W. Wilson and Y.T. Gui, Pulsed electromagnetic methods for defect detection and characterisation, NDT $\&$ E International, 40(4), 2007, 275-283.

\section{Biographies}

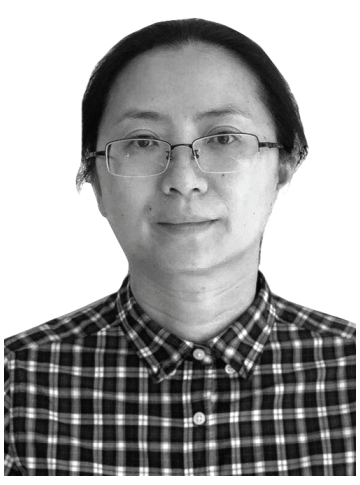

Jia Jia received her master's degree from Zhejiang University. Currently, she is a teacher at Zhejiang Institute of Communications and mainly engaged in the research and analysis of road and bridge teaching. 


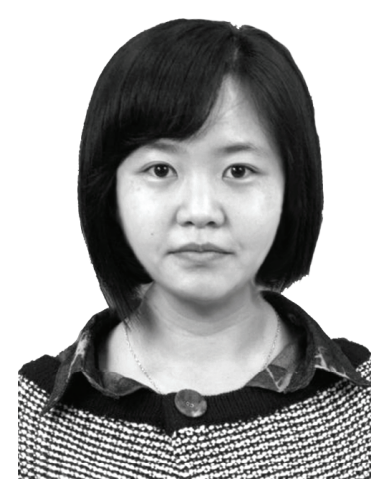

Jun Cheng received the master's degree in Bridge Engineering from Chongqing Jiaotong University, Chongqing, China. Currently, she is a teacher at Chongqing Real Estate College. Her research interests include bridge strengthening, construction materials, and non-destructive testing.

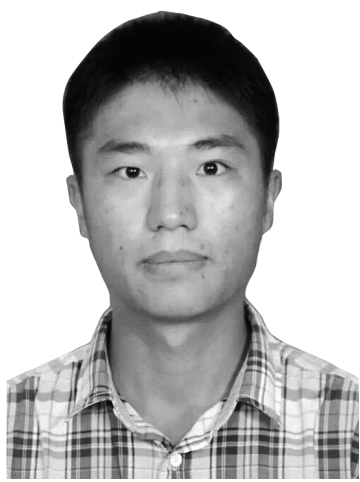

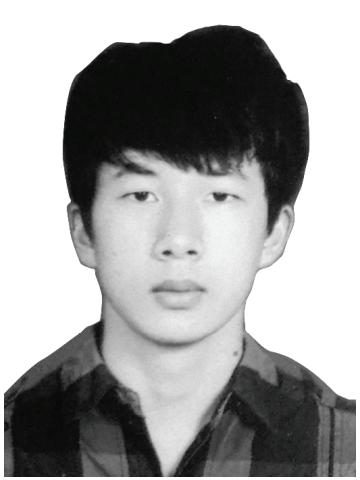

Senhua Zhang is studying for a masterate in Chongqing Jiaotong University, Chongqing, China. His research interests include bridge strengthening, construction materials, and health monitoring.

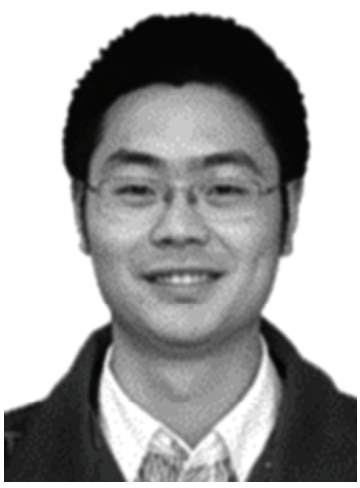

Xiaogang $\mathrm{Li}$ graduated from Chongqing Jiaotong University. Currently, he is studying for a doctorate in Chongqing Jiaotong University, mainly engaged in bridge strengthening and health monitoring. 